\title{
SOURCE ABBREVIATIONS
}

Abreu Gómez

Aguilar Camín

Agraz, 1958

Album de la Paz

Almada

Almada, 1968

Almada, Colima

Almanaque de Aguas

Almanaque de Chiapas

Almanaque de Oaxaca

Almanaque de Puebla

Almanaque de Sonora

Almanaque de Tabasco
Ermilo Abreu Gómez, Sala de retratos (Mexico City: Secretaría de Educación Pública, 1947)

Héctor Aguilar Camín, La frontera nómada:

Sonora y la revolución mexicana (Mexico City: Siglo XXI, 1977)

Gabriel Agraz García de Alba, Ofrenda a México (Guadalajara, 1958)

Ireneo Paz, Album de la paz y el trabajo (Mexico City: Ireneo Paz, 1911)

Francisco R. Almada, Gobernadores del estado de Chihuahua (Mexico City: Cámara de Diputados, 1950)

Francisco R. Almada, Diccionario de historia, geografía y biografía chihuahuenses (Chihuahua: 1968)

Francisco R. Almada, Diccionario de historia, geografía y biografía de Colima (Colima, 1939)

Almanaque de Aguascalientes (Mexico City: Almanaque de México, 1982) Almanaque de Chiapas (Mexico City: Almanaque de México, 1982) Almanaque de Oaxaca (Mexico City: Almanaque de México, 1982) Almanaque de Puebla (Mexico City: Almanaque de México, 1982) Almanaque de Sonora (Mexico City: Almanaque de México, 1982) Almanaque de Tabasco (Mexico City: Almanaque de México, 1982) 
Alvarez Coral

Amigos

Andrade

Aranda Pamplona

Armijo

Azpurua

Balmori

Blue Book

Bojórquez

Bonavit

Bulnes

Bustillos

Carrasco Puente

Carreño

C de D

C de S

Chiapas

Cockcroft
Juan Alvarez Coral, Galería de gobernadores de Quintana Roo (Mexico City: Gobierno de Quintana Roo, 1972)

Sociedad de Amigos del Libro Mexicano, Directorio de escritorios mexicanos (Mexico City, 1956)

Cayetano Andrade, Antología de escritores nicolaitas, 1540-1940 (Mexico City:

Vanguardia Nicolaita, 1941)

Hugo Aranda Pamplona, Biobibliografía de los escritores del estado de México (Mexico City: UNAM, 1978)

Raymundo Armijo Rodríguez, Este día en la historia (Mexico City: Editorial Letras, 1968)

Ramón Azpurua, Biografías de hombres notables de Hispano América (Mexico City, 1877)

Diana Balmori et al., Notable Family

Networks in Latin America (Chicago:

University of Chicago Press, 1984)

Blue Book of Mexico (Mexico City, 1901) Juan de Dios Bojórquez, Forjadores de la revolución mexicana (Mexico City, 1960) Julián Bonavit, Fragmentos de la historia del Colegio Primitivo (Morelia, 1910)

Pepe Bulnes, Gobernantes de Tabasco (Mexico City, 1978)

Antonio Bustillos Carrillo, Apuntes históricos y biográficos (Mexico City, 1953)

Rafael Carrasco Puente, Datos históricos iconografía de la educación en México (Mexico City: Secretaría de Educación Pública, 1960)

Alberto María Carreño, La Academia Mexicana Correspondiente de la Española, 1875-1945 (Mexico City: Secretaría de Educación Pública, 1946)

Cámara de Diputados, Memoria, each legislature, date, 1880-1988

Cámara de Senadores, Memoria, each legislature, date 1880-1988 Chiapas, 1925 (no publisher or date) James D. Cockcroft, Intellectual Precursors of the Mexican Revolution, 1900-13 (Austin: University of Texas Press, 1968) 
Colliman

Cordero

Covarrubias

Covarrubias, I-IV

Cruzado

Cuéllar Valdés

Cuevas

CyT

Dávila

DBGM, 1984

DBM68

DGF50

DGF51

DGF56

\section{de Parrodi}

Dicc mich

Dir social

DP70

DPE61

Dulles

EBW
Daniel A. Moreno, Colliman, 2 Vols. (Mexico City, 1952-1953)

Enrique Cordero y Torres, Diccionario General de Puebla (Puebla, 1958)

Ricardo Covarrubias, Los 67 gobernadores de México independiente (Monterrey, 1952)

Ricardo Covarrubias, Las calles de Monterrey, 4 Vols. (Monterrey, 1970)

Manuel Cruzado, Bibliografía jurídica mexicana (Mexico City: Oficina de Estampillas, 1905)

Pablo Cuéllar Valdés, Historia del estado de Coahuila (Saltillo: Universidad Autónoma de Coahuila, 1979|

Gabriel Cuevas, El glorioso Colegio Militar mexicano en un siglo 1824-1924 (Mexico City, 1937)

Enrique Cordero y Torres, Diccionario biográfico de Puebla, 2 Vols. (Mexico City, 1972) José María Dávila, El ejército de la revolución (Mexico City: Slyse, 1938)

Diccionario biográfico del gobierno mexicano (Mexico City: Presidencia de la República, 1984)

Diccionario biográfico de México, 1966-68

(Monterrey: Editorial Revesa, 1968)

Mexico, Dirección Técnica de Organización, Directorio del gobierno federal, 1950

Mexico, Dirección Técnica de Organización, Directorio del gobierno federal, 1951

México, Dirección Técnica de Organización, Directorio del gobierno federal, 1956

Enriqueta de Parrodi, Sonora, hombres y paisajes (Mexico City: Editorial Pafim, 1941) Jesús Romero Flores, Diccionario michoacano de historia y geografía (Morelia, 1960)

Directorio Social (Mexico City, 1935)

Diccionario Porrúa (Mexico City: Editorial Porrúa, 1970)

Directorio del poder ejecutivo, 1961 (Mexico City, 1961)

John W. F. Dulles, Yesterday in Mexico (Austin: University of Texas Press, 1961) Biographical Encyclopedia of the World (New York: Institute for Research in Biography, 1946) 
Enc Mex

Enc de Yucatán

Enc Reg Coah

Estrada

Excélsior

FSRE

Func

Fusco

García Cubas

García de Alba

García Purón

García Rivas

Garrido

Ghigliazza

Godoy

Gómez

González Dávila

González de Cossío
Enciclopedia de México (Mexico, various years)

Carlos A. Echanove Trujillo, Enciclopedia yucatense (Mexico City: Gobierno de Yucatán, 1944-1947)

Diccionario enciclopédico regional del estado de Coahuila (Mexico: Fernández, 1966)

Genaro Estrada, Poetas nuevos de México (Mexico City: Ediciones Porrúa, 1916) Excélsior (Mexico City) Funcionarios de la Secretaría de Relaciones Exteriores, desde el año de 1821 a 1940 (Mexico City: SRE, 1940)

Sergio Serra Domínguez and Roberto Martínez Barreda, México y sus funcionarios (Mexico: Litográfico Cárdenas, 1959)

Federico M. Fusco and Félix M. Iglesias, Los hombres que rodean al señor general Porfirio Díaz (Mexico City: La Paz Pública, 1896) Antonio García Cubas, Diccionario geográfico, histórico y biográfico de los Estado Unidos Mexicanos (Mexico City: Antigua Imprenta de Murguía, 1888-1891)

Gabriel Agraz García de Alba, Jalisco y sus hombres (Guadalajara, 1958)

Manuel García Puron, México y sus gobernantes, biografías (Mexico: Porrúa, 1964) Heriberto García Rivas, 150 biografías de mexicanos ilustres (Mexico City: Diana, 1964) Luis J. Garrido, El partido de la revolución institucionalizada (Mexico City: Siglo XXI, 1982)

Manuel Mestre Ghigliazza, Apuntes para una relación de los gobernantes de Tabasco (Mérida, 1984)

José F. Godoy, Enciclopedia biográfica de contemporáneos (Washington, D.C.: Globe Printing Office, 1898)

Marte R. Gómez, Biografías de agrónomos (Mexico City: Escuela Nacional de Agricultura, 1976)

Amado González Dávila, Diccionario geográfico, histórico, biográfico y estadístico del estado de Sinaloa (Culiacán, 1959)

Francisco González de Cossío, Xalapa (Mexico City, 1957) 
González de la Garza

Gordillo y Ortiz

Grimaldo

Gruening

Guerra

HA

Holms

Hombres prominentes

Ind biog

Illescas

Inguíñiz

Ipiña de Corsi

Ipiña de Corsi, 1956

Iturribarría

JSH

Justicia

Langston

Lanuza

Leight

Libro azul
Rodolfo González de la Garza, Mil familias de Tamaulipas, Nuevo León, Coahuila y Texas, 2 Vols. (Mexico City, 1980)

Diccionario biográfico de Chiapas (Mexico City: Costa Amic, 1977)

Isaac Grimaldo, Apéndice de mexicanos distinguidos (Mexico City, 1946)

Ernest Gruening, Mexico and Its Heritage

(New York: D. Appleton-Century, 1928)

F. X. Guerra, Le Mexique: De l'Ancien Régime a la Revolution, 2 Vols. (Paris: L'Harmattan, 1985)

Hispano Americano or Tiempo

P. G. Holms, The Directory of Agencies, Mines and Haciendas, 1905-06 (Mexico City: American Book and Printing Company, 1905) Ireneo Paz, Hombres prominentes de México (Mexico City: La Patria, 1888)

Arturo Blancas and Tomás L. Vidrio, Indice biográfico de la XLIII Legislatura Federal (Mexico City, 1956)

Francisco R. Illescas and Juan Bartolo Hernández, Escritores veracruzanos, reseñas biográfica-antológica (Veracruz, 1945)

Juan B. Inguíniz, Bibliografía biográfica mexicana (Mexico City: UNAM, 1969)

Matilde Ipiña de Corsi, La familia Hernández Soto de San Luis Potosí (San Luis Potosí, 1966) Matilde Ipiña de Corsi, Cuatro grandes dinastías en los descendientes de los hermanos Fernández de Lima y Barragán (San Luis Potosí, 1956)

Jorge Fernando Iturribarría, La generación oaxaqueña del 57 (Mexico City, 1956)

Biografías de amigos y conocidos (Mexico: Cuadernos Americanos, 1980)

Justicia (Mexico City legal review) William S. Langston, "Coahuila in the Porfiriato, 1893-1911," Unpublished Ph.D. dissertation, Tulane University, 1980.

Agustín Lanuza, Historia del Colegio de Guanajuato (Guanajuato, 1924)

Hugo Leight, Las calles de Puebla (Puebla, 1967)

Rosario Sansores Pren, Libro azul de la sociedad mexicana (Mexico City, 1946) 
Libro de oro

Linajes

López

López, Héctor

López González

Lutrell

MAH

Márquez

Martínez Alomía

Mata Torres

Medina, No. 20

Mellado

Mestre

México y sus hombres Meyer, No. 12

Montejano

Morales Jiménez

Moreno

Mundo Lo
H. Ruiz Sandoval, Jr., El libro de oro de México (Mexico City, 1967-1968)

Torsten Dahl, Linajes en México (Mexico

City: Casa Editora de Genealogía Ibero Americana, 1967)

José López Escalera, Diccionario biográfico y de historia de México (Mexico City: Editorial del Magistrado, 1964)

Héctor F. López, Diccionario geográfico, histórico, biográfico y lingüístico del estado de Guerrero (Mexico City: Pluma y Lápiz, 1942)

Valentín López González, Los compañeros de Zapata (Morelos, 1980)

Estelle Lutrell, Mexican Writers (Tucson, 1920)

Academia Mexicana de Historia, Memorias (Mexico City, various years)

Joaquín Márquez Montiel, Hombres célebres de Chihuahua (Mexico City: Editorial Jus, 1953)

Gustavo Martínez Alomía, Historiadores de Yucatán (Campeche: El Fénix, 1906)

Ramón Mata Torres, Personajes ilustres de Jalisco (Mexico City, 1978)

Luis Medina, Historia de la revolución mexicana, periodo 1940-1952, Vol. 20 (Mexico: El Colegio de Mexico, 1979)

Guillermo Mellado, Tres etapas políticas de Don Venustiano (Mexico City, 1916)

Manuel Mestre Ghigliazza, Efemérides biográficas (Mexico City: Antigua Librería Robredo, 1945)

México y sus hombres (Mexico City, 1905) Lorenzo Meyer, et al., Historia de la revolución mexicana periodo 1928-1934, Vol. 12 (Mexico City: Colegio de Mexico, 1978) Rafael Montejano y Aguinaga, Biobibliografía de los escritores de San Luis Potosí (Mexico City: UNAM, 1979|

Alberto Morales Jiménez, Hombres de la revolución mexicana (Mexico City: Talleres Gráficos de la Nación, 1960)

Daniel Moreno, Colima y sus gobernadores (Mexico City, Ediciones Studium, 1953) Sarah de Mundo Lo, Index to Spanish Ameri- 
Naranjo

Nakayama

NYT

O'Campo

Ortega

Palavicini

Parrodi

Pasquel

Pasquel, 1972

Pasquel, Jalapa

Pavía

Pavía, breves

Pavía, judges

Peña

Peral

Peral 47

Pérez Galaz

Pérez López can Collective Biography, Vol. 2 Mexico (New York: G. K. Hall, 1982)

Francisco Naranjo, Diccionario biográfico revolucionario (Mexico City: Editorial Cosmos, 1935)

Antonio Nakayama, Sinaloa, el drama y sus actores (Mexico City: INAH, 1975)

The New York Times

Aurora O'Campo and Ernesto Prado Velázquez, Diccionario de escritores mexicanos (Mexico City: UNAM, 1967)

Ricardo Ortega, Pérez Gallardo, Historia genealógica de las familias más antiguas de México (Mexico City: A. Carranza, 1908) Félix F. Palavicini, Grandes de México (Mexico City: Sociedad Bolivariana, 1948)

Enriqueta de Parrodi, Sonora, hombres y paisajes (Mexico City: Editorial Pafím, 1941) Leonardo Pasquel, Educadores veracruzanos (Mexico City: Editorial Citlaltepetl, 1983) Leonardo Pasquel, La generación liberal veracruzana (Veracruz: Editorial Citlaltepetl, 1972) Leonardo Pasquel, Xalapeños distinguidos (Jalapa: Editorial Citlaltepetl, 1983)

Lázaro Pavía, Los estados y sus gobernantes (Mexico City: Escalerillas, 1890) Lázaro Pavía, Breves apuntes biográficos de los miembros más notables del ramo de hacienda de la república mexicana (Mexico City: Dublán, 1895)

Lázaro Pavía, Apuntes biográficos de los miembros más distinguidos del poder judicial (Mexico City: Barroso, 1893)

Antonio de la Peña y Peña, Vidas y tiempos (Havana: El Renacimiento, 1915) Miguel Angel Peral, Diccionario biográfico mexicano (Mexico City: Editorial PAC, 1945?) Miguel Angel Peral, Diccionario biográfico mexicano suplemento (Mexico: Editorial PAC, 1947)

Juan de Dios Pérez Galaz, Diccionario geográfico e histórico de Campeche (Campeche, 1944)

Abraham Pérez López, Diccionario biográfico hidalguense (Mexico: Imprenta Unión, 1979) 
Política

Puente

QesQ

QesQM

Quirós

Ramírez

Rev de Ejer

Rice

Romero Aceves

Romero Flores

Romo

Rouaix

Sánchez García

Santamaría

Sec of War

Serrano

Siempre

Sierra

Siliceo

Sociedad
Política

Ramón Puente, La dictadura, la revolución y sus hombres (Mexico City, 1938)

Carlos Morales Díaz, Quién es quien en la nomenclatura de la ciudad de México (Mexico

City: Costa Amic, 1971)

Quién es cada quien en Monterrey (Monterrey, 1952)

Roberto Quirós Martínez, El momento actual (Mexico City, 1924)

Alfonso Francisco Ramírez, Hombres notables y monumentos coloniales de Oaxaca (Mexico City, 1948)

Revista de Ejército y Fuerza Aérea Jacqueline Ann Rice, "The Porfirian Political Elite: Life Patterns of the Delegates to the 1892 Unión Liberal Convention," Unpublished Ph.D. dissertation, UCLA, 1979. Ricardo Romero Aceves, Maestros colimenses (Mexico City: Costa Amic, 1975)

Jesús Romero Flores, Maestros y amigos (Mexico City: Costa Amic, 1971)

Joaquín Romo de Vivar y Torres, Guadalajara (Guadalajara: Banco Industrial de Jalisco, 1964) Pastor Rouaix, Diccionario geográfico, histórico y biográfico del estado de Durango (Mexico City: Instituto Pan Americano de Geografía e Historia, 1946)

Alfonso Sánchez García, Historia elemental del estado de México (Toluca: Gobierno del Estado, 1983)

Francisco J. Santamaría, Semblanzas tabasqueñas (Mexico, 1946)

México, Secretaría de Guerra y Marina, Escalafón general de ejército (Mexico City, 1902, 1911, 1914)

T. F. Serrano, Episodios de la revolución en México (El Paso: Modern Printing, 1911) Siempre (Mexico City)

Carlos J. Sierra, Historia de la administración hacendaria en México (Mexico City: Secretaría de Hacienda Pública, 1970)

Rosario Siliceo Ambia, Perfiles de gloria (Puebla: Centro de Estudios Históricos, 1969) México, Sociedad Mexicana de Geografía y Estadística, Boletín de la Sociedad Mexicana de 
Sosa

Teixidor

Torre Villar

Torres Martínez

UNAM, ENP

UNAM, law

UNAM, med

UTEHA

Valdés Acosta

Velasco

Velásquez Bringas

Villa, 1937

Villaseñor

WB48

WB54

Wright

WWLA35

WWLA40

WWM45

\section{Geografía y Estadística}

Francisco Sosa, Biografías de mexicanos distinguidos (Mexico City: Secretaría de Fomento, 1884)

Felipe Teixidor, Exlibris y bibliotecas en México (Mexico City: Secretaría de Relaciones Exteriores, 1931)

Ernesto de la Torre Villar, Mexicanos ilustres, Vol. 2 (Mexico City: Jus, 1979)

Gonzalo Torres Martínez, Los Torres de Jaen en México (Mexico City: Editorial Jus, 1975) Inscripciones (UNAM, National Preparatory School, official registration records, all years) Inscripciones (UNAM, National School of Law, official registration records, all years) Inscripciones (UNAM, National School of Medicine, official registration records, all years)

Diccionario Enciclopedia UTEHA (Mexico: UTEHA, 1950)

José María Valdés Acosta, $A$ través de las centurias (Mérida, 1931)

Alfonso Luis Velasco, Porfirio Díaz y su gabinete, estudios biográficos (Mexico City: Editores, 1889|

Esperanza Velásquez Bringas and Rafael Heliodoro Valle, Indice de escritores (Mexico City: Herrero, 1928)

Eduardo W. Villa, Educadores Sonorenses (Mexico City, 1937)

Ramiro Villaseñor y Villaseñor, Bibliografía general de Jalisco (Guadalajara: Gobierno del Estado, 1957)

World Biography (New York: Institute for Research in Biography, 1948)

World Biography (New York: Institute for Research in Biography, 1954)

Laureana Wright de Kleinhans, Mujeres notables mexicanas (Mexico City: Económica, 1910)

Percy A. Martin, Who's Who in Latin America (Stanford: Stanford University Press, 1935) Percy A. Martin, Who's Who in Latin America (Stanford: Stanford University Press, 1940) Percy A. Martin, Who's Who in Latin America (Stanford: Stanford University Press, 1945) 
THIS PAGE INTENTIONALLY LEFT BLANK 
Mexican Political Biographies, 1884-1935 
THIS PAGE INTENTIONALLY LEFT BLANK 